\title{
ANTI-JO-1 ANTISYNTHETASE SYNDROME AND SYSTEMIC LUPUS ERYTHEMATOSUS OVERLAP SYNDROME: A CASE REPORT
}

\author{
Thiago Quadrante Freitas ${ }^{1, \star}$, André Silva Francoํㅡㄹ Emily Figueiredo Neves Yuki ${ }^{1}$, Samuel Katsuyuki Shinjo ${ }^{1}$ \\ 1. Hospital das Clinicas HCFMUSP, Faculdade de Medicina, Universidade de São Paulo, São Paulo (SP), Brazil. \\ *Corresponding author: thiago.f@fm.usp.br
}

\section{BACKGROUND}

Systemic lupus erythematosus (SLE) may coexist with immune-mediated myopathies in roughly $7 \%$ of the cases. Most of these patients have mild or no muscle symptoms and they usually follow SLE activity. However, herein we report a patient with stable SLE who developed significant muscle weakness and high serum levels of muscle enzymes, being characterized a posteriori as antisynthetase syndrome.

\section{CASE REPORT}

A 35-year-old black woman presented in May 2019 with hand arthralgias, fever and weight loss, lymphopenia, serositis, livedo reticularis and venous and arterial thrombosis requiring transtibial amputation of the right lower limb. Her autoimmunity panel showed a nuclear fine speckled and cytoplasmic fine speckled antinuclear antibody pattern with positive anti-Ro and anti-P autoantibodies; IgM anticardiolipin and lupus anticoagulant were positive on only one occasion. Systemic lupus erythematosus was diagnosed, and she was treated with methylprednisolone pulse therapy, hydroxychloroquine, methotrexate, and warfarin with a good outcome. After 15 months, the patient had no signs of SLE activity and was using only hydroxychloroquine and methotrexate. However, serum levels of creatine phosphokinase (CPK) increased from 366 to $2580 \mathrm{U} / \mathrm{L}$. Two months later, she presented with diffuse myalgia and proximal muscle weakness (muscle strength grade IV), with CPK rising to $15299 \mathrm{U} / \mathrm{L}$. The patient was readmitted for investigation. Skeletal muscle magnetic resonance showed shoulder and pelvic girdle muscles with edema, in addition to thickening and edema of muscular fasciae. Muscle biopsy predominantly showed necrosis with macrophagia and mild CD4 and CD8 endomysial inflammatory infiltrates, with moderate MHC-I staining. A chest computed tomography revealed nonspecific interstitial pneumonia, and laboratory workup with positive anti-Jo-1 autoantibody. With this hypothesis of anti-Jo- 1 antisynthetase syndrome, the patient received methylprednisolone pulse therapy $(3 \mathrm{~g})$, and methotrexate was reintroduced with good clinical and laboratory outcomes.

\section{CONCLUSION}

To the best of our knowledge, this is the first case of anti-Jo-1 antisynthetase syndrome and SLE overlap syndrome, which should be remembered and considered in our clinical practice.

\section{KEYWORDS}

Systemic lupus erythematosus, Antisynthetase syndrome, Immune-mediated myopathies. 